\title{
Risk factors for sporadic salmonellosis: a systematic review and meta-analysis
}

\author{
Laurent Guillier ${ }^{\mathrm{a}, *}$, Anne Thébault ${ }^{\mathrm{a}}$, Philippe Fravalo ${ }^{\mathrm{b}, \mathrm{c}}$, Lapo Mughini-Gras ${ }^{\mathrm{d}, \mathrm{e}}$, \\ Nathalie Jourdan-da Silva ${ }^{\mathrm{f}}$, Julie David ${ }^{\mathrm{g}}$, Pauline Kooh ${ }^{\mathrm{a}}$, Vasco Cadavez ${ }^{\mathrm{h}}$, Ursula Gonzales- \\ Barron ${ }^{\mathrm{h}}$ \\ ${ }^{a}$ Risk Assessment Department, French Agency for Food, Environmental and Occupational Health \& Safety (Anses), 14 rue Pierre et Marie Curie Maisons-Alfort, France \\ ${ }^{\mathrm{b}}$ Veterinary School, University of Montreal, 3200 Sicotte Saint-Hyacinthe J2S7C6 Qc Canada \\ ${ }^{\mathrm{c}}$ Cnam, 2 Rue Camille Guérin, 22400 Ploufragan, France \\ ${ }^{\mathrm{d}}$ National Institute for Public Health and the Environment (RIVM), Centre for Infectious Disease Control (CIb), PO Box 1 - 3720 BA Bilthoven, the Netherlands \\ ${ }^{\mathrm{e}}$ Utrecht University, Faculty of Veterinary Medicine, Yalelaan 2, De Uithof - 3584 CL Utrecht, the Netherlands \\ ${ }^{\mathrm{f}}$ Santé Publique France, 12 Rue du Val d'Osne, Allée Vacassy, 94410 Saint-Maurice, France \\ ${ }^{g}$ Anses, Ploufragan-Plouzané laboratory, Beaucemaine, 22440 Ploufragan, France \\ ${ }^{\text {h }}$ Centro de Investigação de Montanha (CIMO), Instituto Politécnico de Bragança, Campus de Santa Apolónia, 5300-253 Bragança, Portugal
}

\section{A R T I C L E I N F O}

\section{Keywords:}

Research synthesis

Case-control studies

Meta-regression

Salmonella

\begin{abstract}
A B S T R A C T
Non-typhoidal Salmonella is an important causative agent of diarrheal illness worldwide. A systematic review and meta-analysis of case-control studies were performed to determine the main risk factors associated with sporadic salmonellosis. Suitable scientific articles published up to 2017 were identified through a systematic literature search and subject to methodological quality assessment. From each study, odds ratios (OR) were extracted or calculated, as well as study characteristics such as population type, design, type of model used and risk factor categorization. Mixed-effects meta-analytical models were adjusted by population type to appropriate data partitions. From 3858 identified references, the quality assessment stage was passed by 62 case-control studies focusing on sporadic salmonellosis which provided 1154 ORs for meta-analysis.

The meta-analysis identified host-specifics factors, travel, environmental, animal and food exposures as significant risk factors of salmonellosis in the mixed population. For the mixed population, foods significantly associated with salmonellosis were eggs and egg products, composite foods, and meat (pork, red meats other than beef and poultry meats). In the child population, the main risk factors were found for person-to-person transmission, recent use of gastric anti-acids or antibiotics, contact with pets and farm animals, environmental, and food vehicles. Breastfeeding was found to be a protective factor for children. The food vehicles identified in children comprised dairy (milk formula), produce, meat and eggs products. Untreated drinking water was a risk factor for the children and mixed population. The result of the meta-analysis conducted at the international level is very important in the context of increasing international trade in foodstuffs and changes in food consumption patterns.
\end{abstract}

\section{Introduction}

Non-typhoid Salmonella enterica subsp. enterica is a major foodborne pathogen that causes mild to moderate self-limiting gastroenteritis (WHO, 2018). Salmonellosis is usually characterized by acute onset of fever, abdominal pain, diarrhea, nausea and sometimes vomiting. The onset of disease symptoms occurs 6-72 hours (usually 12-36 hours) after the ingestion of Salmonella, and the illness lasts 2-7 days (WHO, 2018). Patients will make a recovery without specific treatment in most cases.

Salmonellosis is the second most frequently reported bacteriologically related zoonosis in many European countries (EFSA and ECDC,

* Corresponding Author. Tel. 33+(0) 149772648

E-mail addresses: laurent.guillier@anses.fr (L. Guillier), philippe.fravalo@lecnam.net (P. Fravalo), lapo.mughini.gras@rivm.nl, lapo.mughini.gras@rivm.nl (L. Mughini-Gras), Nathalie.JOURDAN-DASILVA@santepubliquefrance.fr (N. Jourdan-da Silva), juliedavidp@hotmail.com (J. David), vcadavez@ipb.pt (V. Cadavez), ubarron@ipb.pt (U. Gonzales-Barron). 
2018). In France, the annual community incidence rate was estimated at 307 cases per 100,000 for salmonellosis (Van Cauteren et al., 2015; Van Cauteren et al., 2017). Microbiological and epidemiological investigations make it possible to identify outbreaks and to trace back the food at the origin of the human cases (Inns et al., 2015; Ung et al., 2019). Although Salmonella outbreaks are identified, a majority of salmonellosis cases is not associated with any known outbreak (Glass et al., 2015) and are classified as sporadic cases. The question of specific food exposures and respective weight for these specific forms arise.

There are several methodological approaches for salmonellosis source attribution (Pires et al., 2010; Pires et al., 2014; Mughini-Gras et al., 2018), and the most appropriate approach depends on the data availability and research question to be addressed. A large variety of approaches has been explored, including microbial subtyping method (David et al., 2013a; David et al., 2013b; Barco et al., 2013; Barco et al., 2015; Mikkelä et al., 2019), genomics approach (Zhang et al., 2019), comparative exposure assessment (Christidis et al., 2020), outbreak data analysis (Pires et al., 2010; Painter et al., 2013; IFSAC (Interagency Food Safety Analytics Collaboration) 2019), or expert elicitation (Havelaar et al., 2008).

Another way of identifying the sources of these sporadic cases is casecontrol studies (Domingues et al., 2012). In case-control studies, the association of cases with various food exposures is usually measured through odds ratios (ORs) (Pires et al., 2009). Meta-analyses of these studies can provide information on exposure pathways of interest (Devleesschauwer et al., 2019). Domingues et al. (2012) conducted a meta-analysis comprising thirty-five Salmonella case-control studies identified through a literature search conducted in February 2008.

The aim of the present study is to perform an updated systematic review of case-control studies for human salmonellosis published internationally up to May 2017 and perform a meta-analysis to synthesize data on factors associated with sporadic Salmonella infection, combining the odds ratio from a selection of relevant studies (Gonzales-Barron et al., 2019).

\section{Methods}

The protocol of the systematic review and the meta-analysis model are described in depth in the methodological paper of this special issue (Gonzales-Barron et al., 2019). The screening criteria followed the Preferred Reporting Items for Systematic Reviews and Meta-analyses (PRISMA).

\subsection{Systematic review}

The keywords were defined attending the review question which is to evaluate the association between a (risk) factor and sporadic salmonellosis risk in a population exposed to it. This review question was identified to have a typical PECO structure (Population, Exposure, Comparator and Outcome as key elements). The literature search was conducted in March 2017 using a combination of keywords related to (1) "Salmonella" OR salmonellosis", (2) "case-control" OR "risk factor" OR "cohort" (3) "infection" OR "disease", joined by the logical connector AND.

Systematic searches using a combination of suitable keywords were conducted using the bibliographic engines Science Direct, PubMed, Scielo, ISI Web of Science and Scopus. The literature search was limited to the English, French, Portuguese and Spanish languages.

Each reference record was screened for relevance for inclusion in the meta-analysis study, and subsequently, the methodological quality of the "candidate" studies was assessed using preset quality criteria. Five criteria for inclusion were related to the definition of disease. First, it had to refer to confirmed humans salmonellosis. The second criterion refers to the study design whereby only case-control studies were considered. The third criterion is the presence of information on cases definition. Finally, the quality and the completeness of statistical analysis were assessed. Primary studies that passed the screening for relevance were marked as having the potential for bias if they failed to meet at least one of the methodological quality assessment criteria. Studies related to $S$. Typhi and Paratyphi were excluded from the review.

Data from primary studies were then extracted using a standardised spreadsheet. Data extracted included the relevant study characteristics (location, period, population, serotype, case definition, design, sample size of the groups, type of model, etc.), the categorized risk factors, the setting, the handling practices and the outcome of the study (Odds Ratio (OR)). A data categorisation scheme was established to hierarchically group the risk factors into travel, host-specific factors and pathways of exposure (see the methodological paper of this issue Gonzales-Barron et al., 2019). The variable "population" was stratified into mixed (adults, or undefined age), and children.

\subsection{Data synthesis}

As described in Gonzales-Barron et al. (2019). The joint meta-analytical data was first described using basic statistics. Next, data was partitioned into subsets of categories of risk factors. Meta-analysis models were then fitted to each of the data partitions or subsets in order to estimate the overall OR for travel, host-specific factors and transmission pathways related to person-to-person transmission, animal contact, environmental and food exposures. The meta-analytical models were fitted separately by population type. For some food classes, the effects of handling (i.e., eating raw, undercooked) and setting (i.e., eating out) on the overall ORs were assessed by calculating the ratio of the mean ORs when food was mishandled (or when food was prepared outside the home) to the base ORs.

The statistical analysis was designed to assess the effect of the geographical region, the study period and the analysis type (univariate/ multivariate) on the result. The objective of the region-specific metaanalysis was to inform the decision on the geographical regions that should be kept for the subsequent pooling of ORs. All meta-analytical models were essentially weighted random-effects linear regression models. Once a meta-analytical model was fitted, influential diagnostics statistics based on Cook's distance were assessed to remove any influential observation originating from studies marked as having potentialfor-bias. Publication bias was assessed by funnel plots and statistical tests investigating the effect of the study sample size on the ORs (Gonzales-Barron et al., 2019). Heterogeneity between studies was assessed by three indicators, the between-study variability $\left(\tau^{2}\right)$, the $\mathrm{QE}$ test investigating residual heterogeneity, the variance of residuals and the intra-class correlation $\mathrm{I}^{2}$ (Gonzales-Barron et al., 2019).

All analyses were conducted in the R software implemented with the metafor package (Viechtbauer, 2010).

Pooled ORs were considered as significant when the lower bound of the $95 \%$ CI was equal or greater than one, except for breastfeeding where the upper bound of the confidence interval had to be below one for it to be deemed as significant (protective).

\section{Results}

Descriptive statistics of the case-control studies

From 3858 identified and analyzed references, 460 passed the relevance screening and 62 passed the quality assessment stage (Fig. 1). Appendix 1 summarizes the 62 bibliographic references. Appendix 2 summarizes the main features (country, population, period, design, model, number of cases/controls, quality and numbers of ORs) of the 62 case-control studies used in this meta-analysis. These case-control studies constitute a set of 1,154 ORs from risk factors (above 0.5 except for breastfeeding).

These published studies were conducted in years spanning from 1987 and 2013 (51.6 \% are after 2000). Most publications concerned Europa (29 publications), North America (25 publications), followed by Oceania 


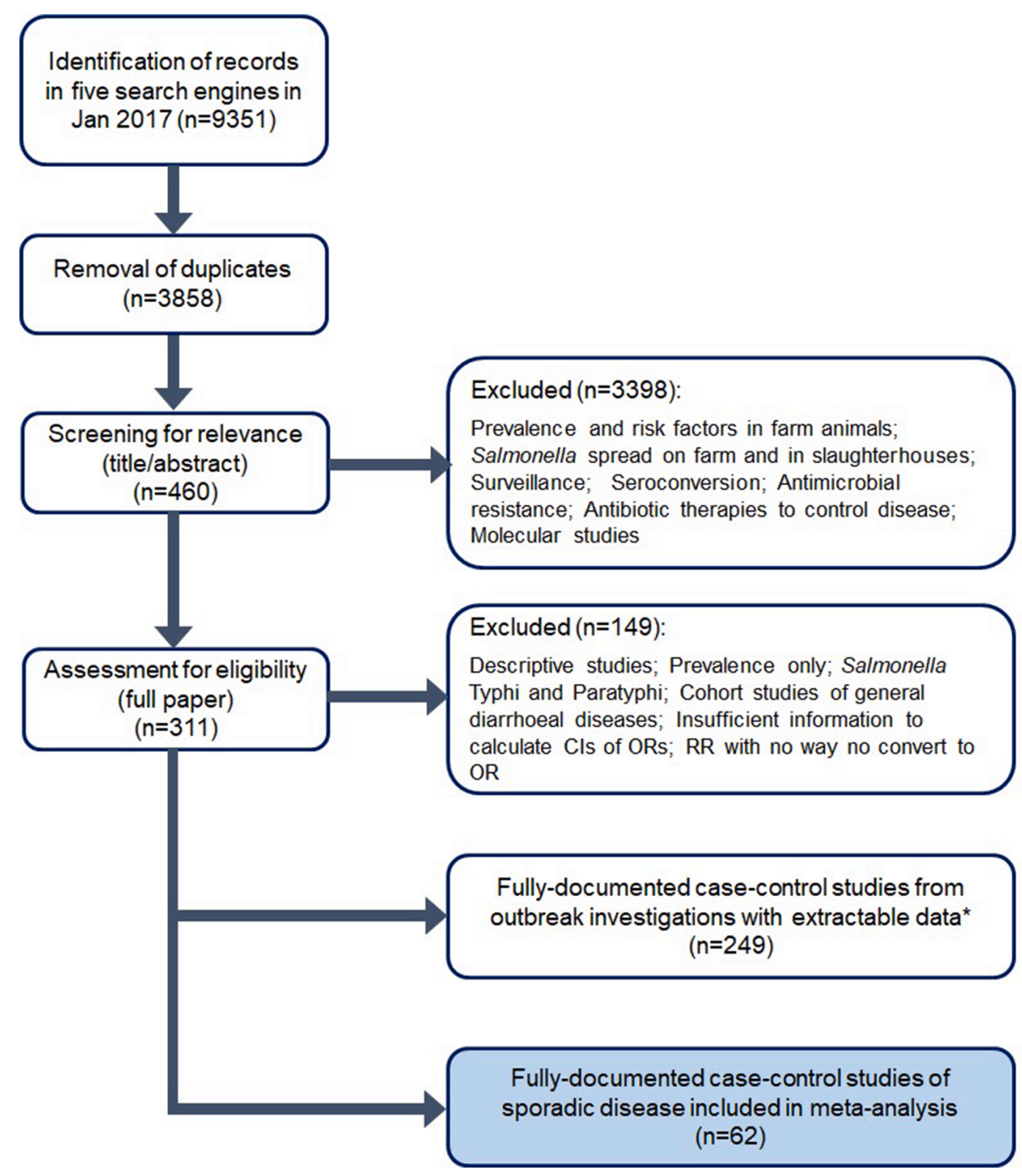

Fig. 1. PRISMA flow chart of the literature search for case-control studies of human salmonellosis.

(5 publications), Asia (2 publications) and South America (1 publication). Europe provided $51 \%$ of the ORs (588/1154) while North America (United States, Canada) is also highly represented with $40 \%$ of ORs (455/1154).

Most studies investigated exposures in the mixed population (adults or non-precise age, 48 publications), followed by child population (16 publications) and susceptible (HIV infected patients) (1 publication) (Appendix 1) (Taconelli et al., 1998). This last publication only contributes to the analysis with one OR concerning antibiotics in hostspecific factors.

Except for one study (Bellido-Blasco et al., 2007), salmonellosis cases were confirmed by isolation of the bacterium. A diversity of serotypes is considered in the 62 studies but the majority (50\%) retained only $S$. Enteritidis and $S$. Typhimurium.

The risk factors studied concerned the following routes of exposure: person-to-person (19 OR), environmental (84 OR), contact with animals (175 OR), and food or handling practices (700 OR). Host-specific factor (122 OR) or travel (54 OR) are also studied in this work.

After methodological quality assessment, 13 case-control studies were marked as being below standards (as example controls have campylobacteriosis) and corresponding ORs were removed from the meta-analysis if sensitivity analysis found an influence on the pooled estimate (Gonzales-Barron et al., 2019).

\subsection{Meta-analysis}

For every data partition, the meta-analysed risk factors are presented in summary tables only when significant (Tables 1, 2 and 3). Nonsignificant results or significant pooled ORs coming from a single study for main risk factors are given in Table 4.

\subsection{Significant risk factors associated with travel, host-related factors, contact with the environment and animals}

Table 1 presents the significant risk factors for these main factors explored. In all geographical regions, except for case-control studies carried out in Asia, travel was a significant risk factor for salmonellosis. Thus, for the estimation of the travel subcategories, the data points from Asia were removed. Both inside and abroad travels were found to be associated with an increased risk of sporadic salmonellosis (pooled OR of $3.61295 \%$ CI [2.839 - 4.593] and 2.195 95\% CI [1.054 - 4.572] respectively. It is worth noticing that before the year 2000, the travel associated OR was significantly higher $(\mathrm{p}=0.005)$.

Several host-specific factors were associated with the risk of salmonellosis. The use of gastric anti-acids or antibiotics had ORs above 3.0 in the mixed population (Table 1) (anti-acids: pooled OR $3.18495 \%$ CI [2.449 - 4.139]). Patients with chronic diseases (i.e. allergies, stomach ulcer, cancer, diabetes, endocrine disease, heart disease, kidney disease, intestinal disorders, alcoholism, autoimmune disease, and blood 
Table 1

Results of the meta-analysis for the main risk factors.

\begin{tabular}{|c|c|c|c|c|c|c|c|c|}
\hline Population & $\begin{array}{l}\text { Geographical } \\
\text { area }\end{array}$ & Risk factor & $\begin{array}{l}\text { Pooled OR } \\
\text { [IC95\%] }\end{array}$ & $\mathbf{N} / \mathbf{n}^{*}$ & $\begin{array}{l}\text { p-value of risk } \\
\text { factor }\end{array}$ & $\begin{array}{l}\text { Publication } \\
\text { bias } \\
\text { p-value }\end{array}$ & $\begin{array}{l}\text { Points } \\
\text { removed } * *\end{array}$ & Heterogeneity analysis**** \\
\hline \multicolumn{9}{|l|}{ Travel } \\
\hline \multirow[t]{2}{*}{ Mixed(y) } & \multirow[t]{2}{*}{ Asia excluded } & Abroad & $\begin{array}{l}3.612[2.839- \\
4.593]\end{array}$ & $\begin{array}{l}18 / \\
47\end{array}$ & $<.0001$ & \multirow[t]{2}{*}{0.408} & \multirow[t]{2}{*}{1} & $\begin{array}{l}\tau^{2}=0.0624 \\
\mathrm{OE}(\mathrm{df}=49)=123.6, \mathrm{p}-\mathrm{val}<\end{array}$ \\
\hline & & Inside & $\begin{array}{l}2.195[1.054- \\
4.572]\end{array}$ & $3 / 3$ & 0.036 & & & $\begin{array}{l}.0001 \\
\mathrm{~s}^{2}=0.925 \\
\mathrm{I}^{2}=6.319 \%\end{array}$ \\
\hline \multicolumn{9}{|c|}{ Host specific } \\
\hline \multirow[t]{4}{*}{$\operatorname{Mixed}(y)$} & \multirow[t]{4}{*}{ All } & Antiacids & $\begin{array}{l}3.184[2.449- \\
4.139]\end{array}$ & $\begin{array}{l}12 / \\
31\end{array}$ & $<.0001$ & \multirow[t]{4}{*}{$<.0001$} & \multirow[t]{4}{*}{0} & \multirow{4}{*}{$\begin{array}{l}\tau^{2}=0.6956 \\
Q E(d f=88)=866.970, p \text {-val } \\
<.0001 \\
\mathrm{~s}^{2}=0.533 \\
\mathrm{I}^{2}=56.626 \%\end{array}$} \\
\hline & & Antibiotics & $\begin{array}{l}3.348[2.657- \\
4.219]\end{array}$ & $\begin{array}{l}14 / \\
30\end{array}$ & $<.0001$ & & & \\
\hline & & Chronic diseases & $\begin{array}{l}3.289[2.449- \\
4.416]\end{array}$ & $8 / 21$ & $<.0001$ & & & \\
\hline & & $\begin{array}{l}\text { Other medical } \\
\text { conditions }\end{array}$ & $\begin{array}{l}4.655[3.458- \\
6.266]\end{array}$ & $5 / 11$ & $<.0001$ & & & \\
\hline \multirow[t]{3}{*}{ Children } & \multirow[t]{3}{*}{ All } & Antiacids & $\begin{array}{l}3.413[1.851- \\
6.296]\end{array}$ & $2 / 3$ & $<.0001$ & \multirow[t]{3}{*}{0.501} & \multirow[t]{3}{*}{0} & \multirow{3}{*}{$\begin{array}{l}\tau^{2}=0.278 \\
Q E(d f=24)=31.053, p-v a l= \\
0.152 \\
\mathrm{~s}^{2}=0.500 \\
\mathrm{I}^{2}=35.724 \%\end{array}$} \\
\hline & & Antibiotics & $\begin{array}{l}2.376[1.618- \\
3.489]\end{array}$ & $6 / 12$ & $<.0001$ & & & \\
\hline & & Breastfeeding & $\begin{array}{l}0.249[0.165- \\
0.377]\end{array}$ & $7 / 12$ & $<.0001$ & & & \\
\hline \multicolumn{9}{|c|}{ Person to person } \\
\hline Mixed & All & & $\begin{array}{l}1.692[1.145- \\
2.500]\end{array}$ & $7 / 10$ & 0.008 & \multirow[t]{2}{*}{0.056} & \multirow[t]{2}{*}{0} & $\begin{array}{l}\tau^{2}=0.557 \\
Q E(d f=17)=58.734, p-v a l<\end{array}$ \\
\hline Children & & & $\begin{array}{l}3.811[1.977- \\
7.348]\end{array}$ & $7 / 9$ & $<.0001$ & & & $\begin{array}{l}.0001 \\
\mathrm{~s}^{2}=0.82 \\
\mathrm{I}^{2}=40.425 \%\end{array}$ \\
\hline \multicolumn{9}{|l|}{ Animals } \\
\hline \multirow[t]{4}{*}{ Mixed } & \multirow[t]{4}{*}{ All } & Pets & $\begin{array}{l}1.471[1.189- \\
1.821]\end{array}$ & $\begin{array}{l}26 / \\
71\end{array}$ & $<.0001$ & \multirow[t]{4}{*}{0.283} & \multirow[t]{4}{*}{1} & \multirow{4}{*}{$\begin{array}{l}\tau^{2}=1.1449 \\
Q E(d f=123)=464.3872, p- \\
\text { val }<.0001 \\
\mathrm{~s}^{2}=0.605 \\
\mathrm{I}^{2}=65.420 \%\end{array}$} \\
\hline & & Exotic (new) pets & $\begin{array}{l}2.619[1.763- \\
3.894]\end{array}$ & $7 / 25$ & $<.0001$ & & & \\
\hline & & Farm animals & $\begin{array}{l}1.937[1.394- \\
2.693]\end{array}$ & $9 / 19$ & 0.0004 & & & \\
\hline & & $\begin{array}{l}\text { Occupational } \\
\text { exposure }\end{array}$ & $\begin{array}{l}1.737[1.175- \\
2.567]\end{array}$ & $5 / 12$ & 0.0056 & & & \\
\hline Children & All & Pets & $\begin{array}{l}3.035[1.938- \\
4.756]\end{array}$ & $9 / 36$ & $<.0001$ & 0.731 & 1 & $\begin{array}{l}\tau^{2}=0.369 \\
Q E(d f=41)=76.2961, p-v a l\end{array}$ \\
\hline & & Farm animals & $\begin{array}{l}2.658[1.431- \\
4.938]\end{array}$ & $2 / 5$ & 0.002 & & & $\begin{array}{l}=0.0007 \\
\mathrm{~s}^{2}=0.540 \\
\mathrm{I}^{2}=40.63 \%\end{array}$ \\
\hline Environmen & & & & & & & & \\
\hline Mixed & All & Recreational water & $\begin{array}{l}2.221[1.339- \\
3.681]\end{array}$ & $9 / 12$ & 0.0020 & 0.088 & 0 & $\begin{array}{l}\tau^{2}=0.7714 \\
Q E(d f=45)=132.6221, p-v a l\end{array}$ \\
\hline & & $\begin{array}{l}\text { Untreated drinking } \\
\text { water }\end{array}$ & $\begin{array}{l}1.753[1.109- \\
2.770]\end{array}$ & $\begin{array}{l}11 / \\
19\end{array}$ & 0.0162 & & & $\begin{array}{l}<.0001 \\
\mathrm{~s}^{2}=0.594\end{array}$ \\
\hline & & Farm environment & $\begin{array}{l}1.900[1.251- \\
2.885]\end{array}$ & $8 / 17$ & 0.0026 & & & $\mathrm{I}^{2}=56.48 \%$ \\
\hline $\begin{array}{l}\text { Children } \\
\text { (at) }\end{array}$ & All & $\begin{array}{l}\text { Untreated drinking } \\
\text { water }\end{array}$ & $\begin{array}{l}2.130[1.285- \\
3.532]\end{array}$ & $5 / 8$ & 0.0034 & 0.199 & 0 & $\begin{array}{l}\tau^{2}=0 \\
\mathrm{QE}(\mathrm{df}=27)=31.5105, \mathrm{p}-\mathrm{val}\end{array}$ \\
\hline & & Daycare attendance & $\begin{array}{l}2.738[1.658- \\
4.522]\end{array}$ & $5 / 10$ & $<.0001$ & & & $\begin{array}{l}=0.2507 \\
\mathrm{~s}^{2}=0.431\end{array}$ \\
\hline & & Playground & $\begin{array}{l}3.055[2.243- \\
4.160]\end{array}$ & $6 / 13$ & $<.0001$ & & & $\mathrm{I}^{2}=0$ \\
\hline Food & & & & & & & & \\
\hline Mixed & All & Meat & $\begin{array}{l}1.489[1.137- \\
1.951]\end{array}$ & $\begin{array}{l}37 / \\
231\end{array}$ & 0.0039 & 0.035 & 8 & $\begin{array}{l}\tau^{2}=0.215 \\
Q E(d f=537)=1971.5989, p-\end{array}$ \\
\hline & & Eggs & $\begin{array}{l}1.774[1.351- \\
2.329]\end{array}$ & $\begin{array}{l}35 / \\
139\end{array}$ & $<.0001$ & & & $\begin{array}{l}\mathrm{val}<.0001 \\
\mathrm{~s}^{2}=0.792\end{array}$ \\
\hline & & Composite & $\begin{array}{l}1.731[1.258- \\
2.381]\end{array}$ & $\begin{array}{l}16 / \\
66\end{array}$ & 0.0008 & & & $\mathrm{I}^{2}=21.34 \%$ \\
\hline Children & All & Meat & $\begin{array}{l}1.794[1.548- \\
2.079]\end{array}$ & $8 / 34$ & $<.0001$ & 0.072 & 2 & $\begin{array}{l}\tau^{2}=0.6705 \\
Q E(d f=76)=93.1415, p-v a l\end{array}$ \\
\hline & & eggs & $\begin{array}{l}1.669[1.295- \\
2.150]\end{array}$ & $7 / 20$ & $<.0001$ & & & $\begin{array}{l}=0.0884 \\
s^{2}=0.482\end{array}$ \\
\hline & & Dairy & $\begin{array}{l}2.828[2.067- \\
3.870]\end{array}$ & $6 / 10$ & $<.0001$ & & & $\mathrm{I}^{2}=58.178$ \\
\hline & & Produce & $\begin{array}{l}2.099[1.169- \\
3.768]\end{array}$ & $2 / 13$ & 0.0130 & & & \\
\hline
\end{tabular}

*N/n Number of studies/number of OR;** points removed by sensitivity analysis, all results are given after removing data concerned; ***Between-study variability $\left(\tau^{2}\right)$, test for residual heterogeneity $(\mathrm{QE})$, variance of residuals $\left(\mathrm{s}^{2}\right)$, intra-class correlation $\left(\mathrm{I}^{2}\right)$. (y): year is significant (before/after 2000) in this model and the estimates are taking this effect into account; (at) analysis type is significant 
Table 2

Results of the meta-analysis on disaggregated risk factors (food).

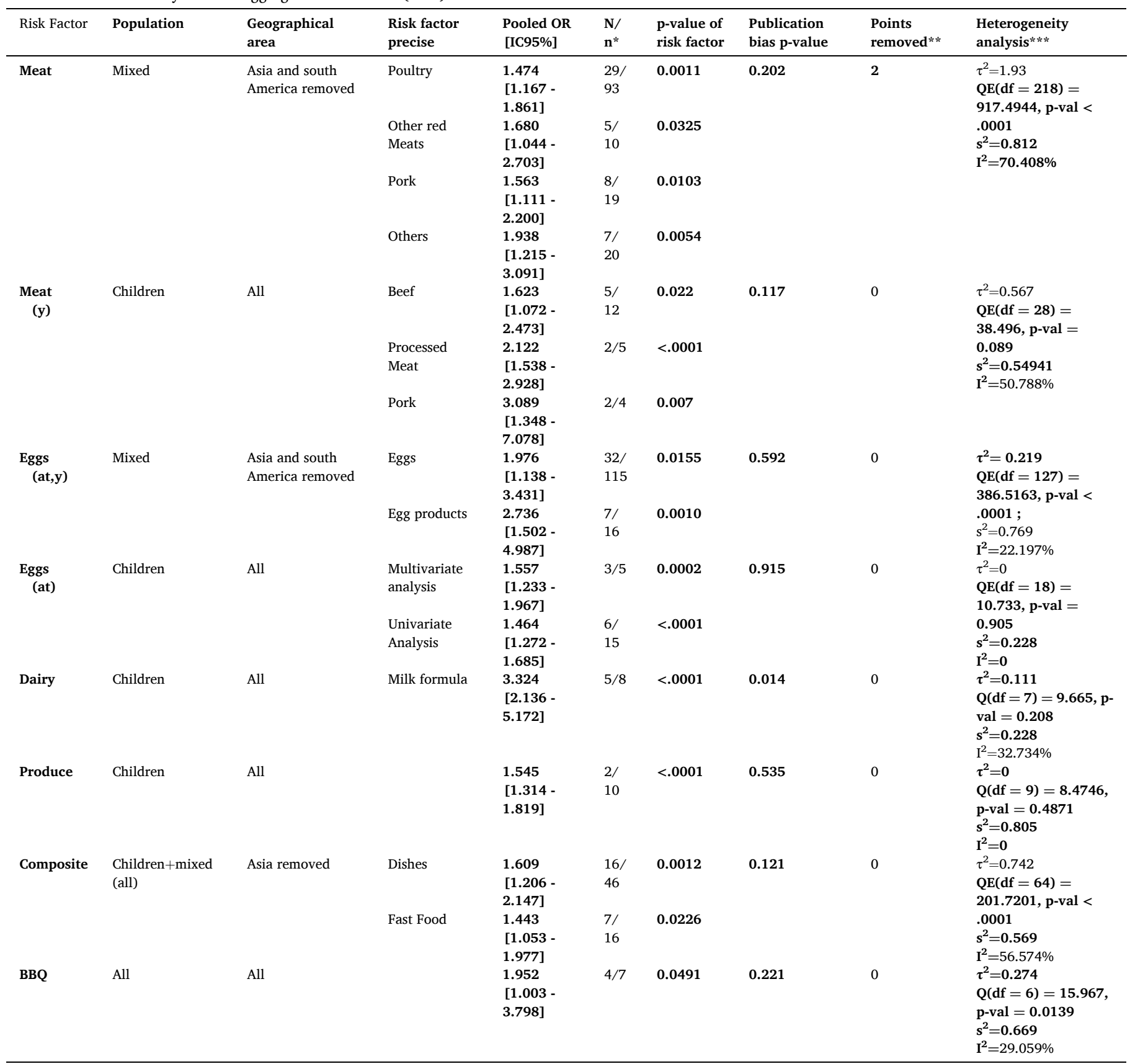

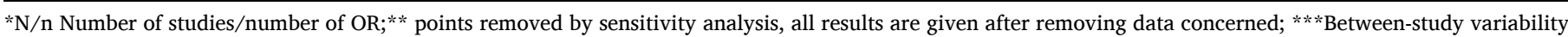

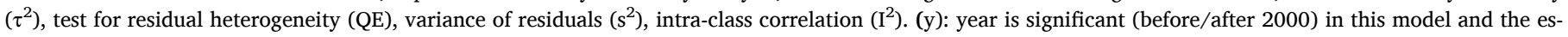
timates are taking this effect into account; (at) analysis type is significant.

disorder) had also significantly higher odds of acquiring salmonellosis (pooled OR=3.289 95\% CI [2.449 - 4.416]). The same level of odds was observed in patients receiving other medications, such as NSAID, corticosteroids, thyroid and hormone drugs (pooled $\mathrm{OR}=4.655$ 95\% CI [3.458 - 6.266]). When focusing on children, the use of anti-acids (pooled OR=3.413 95\% CI $[1.851-6.296]$ ) and antibiotics (pooled $\mathrm{OR}=2.376$ 95\% CI [1.618 - 3.489]) produced a similar degree of salmonellosis risk. In the population of infants, breastfeeding was proven to be protective against Salmonella infection. Combining the results from seven case-control studies, 747 infants of age lower than 2 years had a pooled OR of $0.24995 \%$ CI [0.165 -0.377] times the odds of becoming ill than those who were not breastfed.

Person-to-person transmission was a significant risk factor for both the mixed population and children, but it was much higher in the latter, with a pooled OR of $1.69295 \%$ CI [1.145 - 2.500] and 3.811 [1.977 7.348], respectively. Person-to-person transmission was described in the mixed population as contact with patient or contact household member with diarrhea (or confirmed cases). For the child population, contacts were described as contact with diarrhea in household or creche with an ill child.

Contact with animals (farm, pet or wild) for children as well as in the mixed population was an exposure pathway widely explored in the different studies (127 ORs for 30 studies in the mixed population, and 44 ORs from 9 studies for children). In the mixed population, the pooled ORs for this route of exposure ranged from 1.471 to 2.619 (contact with pets, on-farm activities, or professional activities related to animals and 
Table 3

Effect of food handling on the pooled OR.

\begin{tabular}{|c|c|c|c|c|c|c|c|c|}
\hline Risk Factor & $\begin{array}{l}\text { Risk factor } \\
\text { precise }\end{array}$ & $\begin{array}{l}\text { Pooled OR } \\
\text { [IC95\%] }\end{array}$ & $\mathbf{N} / \mathbf{n}^{*}$ & $\begin{array}{l}\text { p-value of } \\
\text { risk factor }\end{array}$ & $\begin{array}{l}\text { OR ratios } \\
\text { and } C I 95 \%\end{array}$ & $\begin{array}{l}\text { Points } \\
\text { removed ** }\end{array}$ & $\begin{array}{l}\text { Publication bias } \\
\text { p-value }\end{array}$ & $\begin{array}{l}\text { Heterogeneity } \\
\text { analysis } * * *\end{array}$ \\
\hline \multirow[t]{3}{*}{ Poultry (at) } & Eating Out & $\begin{array}{l}3.038[1.965 \\
-4.697]\end{array}$ & $12 / 24$ & $<.0001$ & $\begin{array}{l}1.961 \\
{[1.583-} \\
2.429]\end{array}$ & 3 & 0.032 & $\begin{array}{l}\tau^{2}=0.124 \\
Q E(d f=102)= \\
243.7902, \text { p-val }<.0001\end{array}$ \\
\hline & Undercooked & $\begin{array}{l}3.986[1.641 \\
-9.681]\end{array}$ & $6 / 8$ & 0.0054 & $\begin{array}{l}2.572 \\
{[1.322} \\
-5.005]\end{array}$ & & & $\begin{array}{l}\mathrm{s}^{2}=0.593 \\
\mathrm{I}^{2}=17.311 \%\end{array}$ \\
\hline & Poultry (base) & $\begin{array}{l}1.549[1.241 \\
-1.934]\end{array}$ & $\begin{array}{l}32 / 82 \\
\text { (ref eating out) } \\
34 / 98 \text { (ref handling) }\end{array}$ & 0.0001 & - & & & \\
\hline \multirow[t]{2}{*}{$\begin{array}{l}\text { Processed } \\
\text { Meat }\end{array}$} & Eating out & $\begin{array}{l}1.919[0.922 \\
-3.994]\end{array}$ & $7 / 13$ & 0.021 & $\begin{array}{l}1.635 \\
{[1.074-} \\
2.487]\end{array}$ & 2 & 0.009 & $\begin{array}{l}\tau^{2}=0.303 \\
Q E(d f=58)=207.632 \\
p-v a l<.0001\end{array}$ \\
\hline & $\begin{array}{l}\text { Processed Meat } \\
\text { (base) }\end{array}$ & $\begin{array}{l}1.174[0.858 \\
-1.606]\end{array}$ & $17 / 48$ & 0.315 & - & & & $\begin{array}{l}\mathrm{s}^{2}=0.715 \\
\mathrm{I}^{2}=29.755 \%\end{array}$ \\
\hline \multirow[t]{4}{*}{$\begin{array}{l}\text { Eggs } \\
\text { (at) }\end{array}$} & Eating Out & $\begin{array}{l}2.575[1.465 \\
-4.527]\end{array}$ & $10 / 29$ & 0.0011 & $\begin{array}{l}1.575 \\
{[1.199-} \\
2.069]\end{array}$ & 3 & 0.787 & $\begin{array}{l}\tau^{2}=0.174 \\
\mathrm{QE}(\mathrm{df}=135)= \\
\text { 423.730, p-val }<.0001\end{array}$ \\
\hline & Undercooked & $\begin{array}{l}2.182[1.388 \\
-3.428]\end{array}$ & $22 / 49$ & 0.0004 & $\begin{array}{l}1.335 \\
{[1.136-} \\
1.567]\end{array}$ & & & $\begin{array}{l}\mathrm{s}^{2}=0.818 \\
\mathrm{I}^{2}=17.574 \%\end{array}$ \\
\hline & Raw & $\begin{array}{l}3.642[1.776 \\
-7.469]\end{array}$ & $10 / 12$ & 0.0002 & $\begin{array}{l}2.228 \\
{[1.454-} \\
3.414]\end{array}$ & & & \\
\hline & Base eggs & $\begin{array}{l}1.635 \\
{[1.221-} \\
2.188]\end{array}$ & $\begin{array}{l}\text { 39/111 (ref setting (ie } \\
\text { home or non specified)) } \\
31 / 79 \text { (ref handling (ie } \\
\text { cooked or non } \\
\text { specified)) }\end{array}$ & 0.0009 & - & & & \\
\hline \multirow[t]{2}{*}{ Pork meat } & Undercooked & $\begin{array}{l}3.039[1.289 \\
-7.163]\end{array}$ & $5 / 12$ & $<.0001$ & $\begin{array}{l}3.578 \\
{[2.132-} \\
6.002]\end{array}$ & 0 & $\mathrm{p}=0.259$ & $\begin{array}{l}\tau^{2}=0.1051 \\
Q E(d f=20)=36.145, p- \\
\text { val }=0.0148 \\
s^{2}=0.440\end{array}$ \\
\hline & Base & $\begin{array}{l}0.849 \\
{[0.605-} \\
1.193]\end{array}$ & $7 / 11$ & 0.3475 & - & & & $\mathrm{I}^{2}=19.274 \%$ \\
\hline \multirow[t]{2}{*}{ Vegetables } & Unwashed & $\begin{array}{l}1.542 \\
{[0.773-} \\
3.073]\end{array}$ & $2 / 4$ & 0.112 & $\begin{array}{l}1.551 \\
{[1.002-} \\
2.399]\end{array}$ & 0 & $\mathrm{p}=0.378$ & $\begin{array}{l}\tau^{2}=0.136 \\
Q E(d f=36)=113.719 \\
\text { p-val }<.0001\end{array}$ \\
\hline & Base & $\begin{array}{l}0.994 \\
{[0.772-} \\
1.281]\end{array}$ & $10 / 27$ & 0.964 & - & & & $\begin{array}{l}\mathrm{s}^{2}=0.477 \\
\mathrm{I}^{2}=22.273 \%\end{array}$ \\
\hline
\end{tabular}

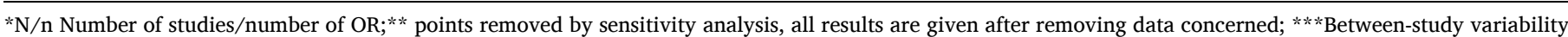

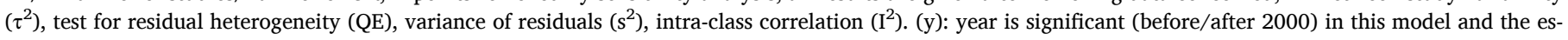
timates are taking this effect into account; (at) analysis type is significant

Table 4

Non significant or isolated ORs (1 publication) in main risk factors.

\begin{tabular}{|c|c|c|c|c|}
\hline Population & $\begin{array}{l}\text { Geographical } \\
\text { area }\end{array}$ & Risk factor & $\begin{array}{l}\text { Pooled OR } \\
\text { [IC95\%] }\end{array}$ & $\mathbf{N} / \mathbf{n}^{*}$ \\
\hline \multicolumn{5}{|l|}{ Travel } \\
\hline Mixed(y) & Asia excluded & Any & $\begin{array}{l}0.909[0.528- \\
1.565]\end{array}$ & $2 / 3$ \\
\hline \multicolumn{5}{|l|}{ Animals } \\
\hline Children & All & $\begin{array}{l}\text { Exotic (new) } \\
\text { pets }\end{array}$ & $\begin{array}{l}1.433 \text { [1.046 - } \\
1.962]]\end{array}$ & $1 / 3$ \\
\hline \multicolumn{5}{|l|}{ Food } \\
\hline \multirow[t]{4}{*}{ Mixed } & All & Produce & $\begin{array}{l}1.228[0.892- \\
1.690]\end{array}$ & $\begin{array}{l}12 / \\
63\end{array}$ \\
\hline & & Seafood & $\begin{array}{l}1.224[0.795 \text { - } \\
1.884]\end{array}$ & $6 / 10$ \\
\hline & & Dairy & $\begin{array}{l}1.187[0.850- \\
1.658]\end{array}$ & $\begin{array}{l}13 / \\
31\end{array}$ \\
\hline & & Beverages & $\begin{array}{l}1.535[0.887- \\
2.655]\end{array}$ & $3 / 4$ \\
\hline Children & All & Seafood & $\begin{array}{l}1.387[0.637 \text { - } \\
3.019]\end{array}$ & $2 / 4$ \\
\hline
\end{tabular}

$\mathrm{N} / \mathrm{n}$ Number of studies/number of $\mathrm{OR}$; if $\mathrm{N}=1$ isolated studies: the result is not considered of significant due to the lack of studies carcasses, and contact with wild animals). Farm environment was also found to be a risk factor in the mixed population (pooled OR $1.90095 \%$ CI [1.251 - 2.885]). For children, the pooled ORs associated with contact with pets and farm animals were $3.03595 \%$ CI [1.938 - 4.756] and $2.65895 \%$ CI [1.431 - 4.938], respectively. When exploring in more detail the pet factor, for both populations, the diversity of animals was important (reptiles, dogs, cats, pets with diarrhea, etc.). The categories of farm animals and wild animals were also very diverse.

For the mixed population, salmonellosis cases occurred more frequently among people exposed to water-related activities (fishing, swimming, sailing, etc.) (pooled OR=2.221 95\% CI [1.339 - 3.681]). Drinking water practice was also significantly associated with increased salmonellosis risk. Within this category, the consumption of untreated water was associated with salmonellosis for the mixed population (pooled OR=1.753 95\% CI [1.109 - 2.770]) and represented a risk factor specifically for children too (pooled OR $=2.13095 \%$ CI [1.285 - 3.532]) (Figure 2). For children, outdoor activities (activities in parks, playground sandboxes, contact with soil, etc.) were strongly associated with salmonellosis (pooled OR=3.055 95\% CI [2.243 - 4.160]). Childcare attendance was significantly associated with salmonellosis (pooled $\mathrm{OR}=2.73895 \% \mathrm{CI}[2.243-4.160]$ ) in children, but strength or ORs were very different in different childcare settings (e.g. school, daycare). 


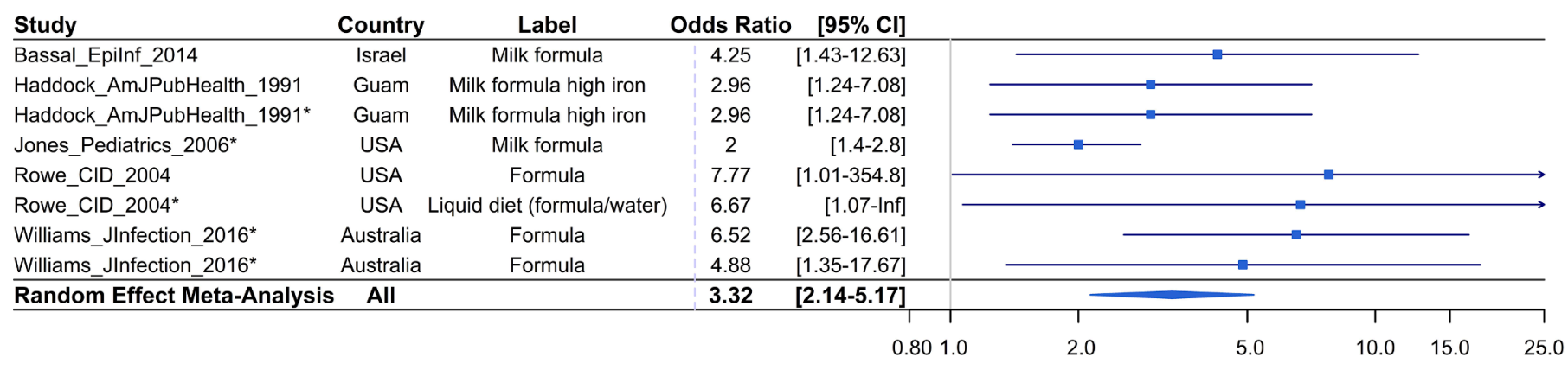

Fig. 2. Forest plot of the associations of salmonellosis with consumption of untreated /not enough treated drinking water in the mixed population (horizontal axis is expressed as OR in logarithm scale).

\subsection{Significant risk factors associated with food-related transmission pathways}

Significant factors associated with food are given in Tables 1, 2 and 3. The studies explored a wide range of foods and food preparation/ consumption practices (e.g. hygiene, cooking, and place of consumption). For the mixed population foods significantly associated with salmonellosis were eggs and egg products (pooled OR=1.774 95\% CI [1.351 - 2.329]), composite foods (pooled OR=1.731 95\% CI [1.258 -2.381]), and meat (pooled OR=1.489 95\% CI [1.137 - 1.951]) (Table 1). Within eggs category, higher pooled OR values were obtained for egg products (pooled OR=2.736 95\% CI [1.502 - 4.987]) compared to eggs (pooled OR=1.976 95\% CI [1.138 - 3.431].

Among meats, poultry (pooled OR=1.474 95\% CI [1.167 - 1.861]), pork (pooled OR $=1.56395 \%$ CI $[1.111-2.200$ ), red meats other than beef (pooled OR=1.680 95\% CI [1.044 - 2.703]) (as lamb, mutton or venison meat), and other meats (pooled OR $=1.938$ 95\%\%CI [1.215 3.091]) were the most important risk factors in the mixed population (Table 2).The category "others" is very heterogeneous containing meats of non-specified origin. The food vehicles identified in children also comprised meat and eggs categories (Table 1). For meat products, pork had the highest pooled OR (pooled OR=3.089 95\% CI [1.348 - 7.078]) followed by processed meat products (pooled $\mathrm{OR}=2.122$ 95\% CI $[1.538$ - 2.928]) and beef (pooled $\mathrm{OR}=1.623$ [1.072 - 2.473]). Produce and dairy were also significantly associated with increased risk of salmonellosis in children (Table 1). The association with dairy, here described as milk formula, was explained by a pooled OR of $3.32495 \%$ CI [2.136 - 5.172] (Figure 3 and Table 2).

The consumption of undercooked pork and poultry meat multiplied the basic ORs by a factor of 3.038 and 3.986, respectively (Table 3). Consumption of raw or undercooked eggs increased the basic ORs by a factor of 3.642 and 2.182, respectively. Out-of-home consumption of poultry, eggs or processed meats increased the basic ORs by factors ranging from 1.5 to 2 (Table 3 ).

For most of the meta-analytical models (models) reported in legends of Tables 1, 2 and 3, the statistical tests indicated the absence of potential significant publication bias is above $5 \%$. An exception is observed in the partitions related to host-specific and food in the mixed population (Table 1), dairy in children (Table 2), and the effect of handling on the consumption of poultry and processed meat (Table 3). For better assessing the publication bias (above 5\%), the funnel plot for those models is given in Figure 4. All of them exhibited asymmetry, as lack of non-significant studies with smaller ORs which could lead to OR overestimation. Moreover, the intraclass correlation $\mathrm{I}^{2}$ were for all partitions below high heterogeneity $(<75 \%)$. However, residual between-study heterogeneity ( $\mathrm{p}$-value often below 0.05 for $\mathrm{Q}$ or $\mathrm{QE}$ ) was observed for the data partitions.

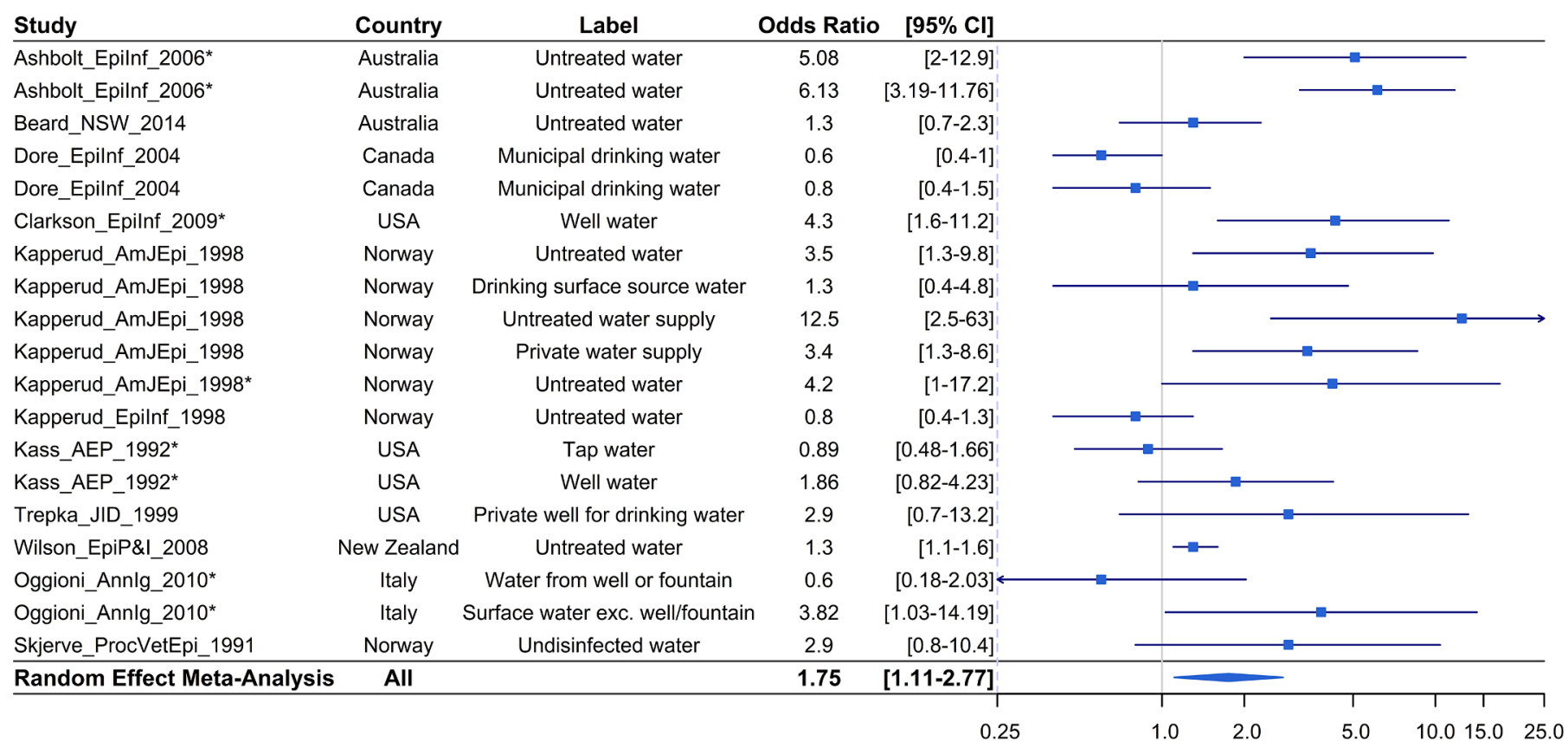

Fig. 3. Forest plot of the associations of salmonellosis with consumption of dairy products in children (OR in logarithm scale). 

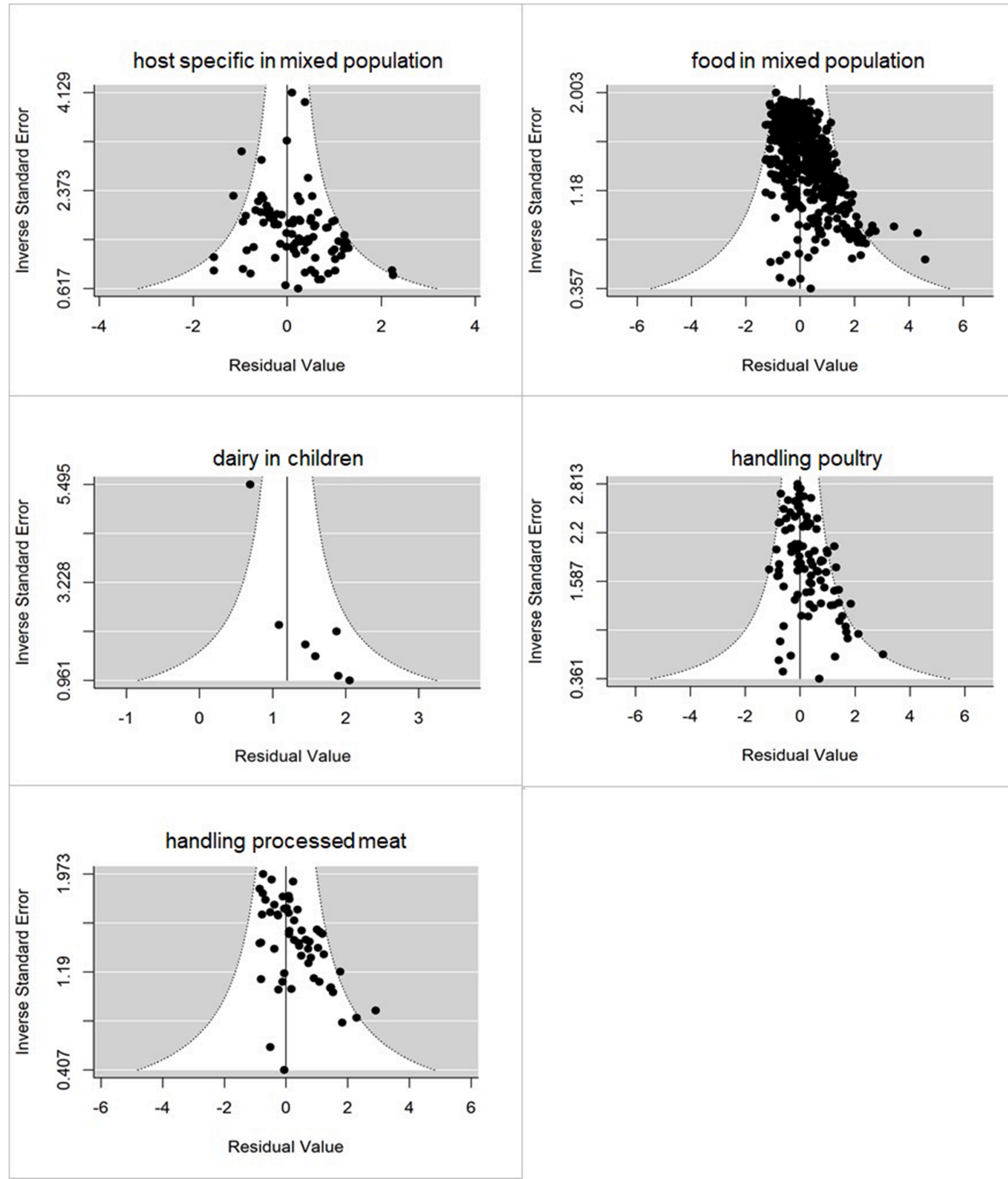

Fig. 4. Funnel plots of studies investigating categorized risk factors (host specific in mixed population, food in mixed population, dairy in children, handling poultry and handling processed meat).

\section{Discussion}

In this study, the aim was to perform an updated systematic review of case-control studies for human salmonellosis and to synthesize data in order to reveal the weight of common factors associated with sporadic
Salmonella infection, among them the relative importance of travel, hostrelated factors, contact with animals and the environment, as well as food-related factors. Based on the results of the meta-analysis, the ORs associated with non-food risk factors (ORs between 2 and 6) were higher than those associated with exposures to main foods categories (ORs 
between 1.4 and 2.9). In agreement with the scientific literature (Domingues et al., 2012; Johnson et al., 2011), the present study identified international travel as a significant risk factor for salmonellosis. Although the number of studies was relatively small, the meta-analysis also allowed for the identification of within-country travel as a significant risk factor; the considered case-control studies concerned large countries, i.e. Australia (Ashbolt and Kirk, 2006) and the US (Passaro et al, 1996; Trepka et al, 1999). Another hypothesis to explain this risk factor is associated with the consumption habits during travelling. Indeed, this risk factor might be a reflection of more frequent eating out practices, which have been also found to be a significant risk factor.

The present meta-analysis also confirmed the findings of Domingues et al. (2012) in the relation to the importance of medication and chronic diseases. Concerning the latter factor, it is worth mentioning that Teunis et al. (2010) did not observe significant differences between the salmonellosis dose-response relations obtained for general and susceptible populations.

The evidenced strong effects of non-food exposures in this metaanalysis suggest that the risk of acquiring salmonellosis is multifactorial and that other sources than the foodborne ones, such as contact with animals (Hoelzer et al., 2011; Lambertini et al., 2016) and environmental waters (Guillier et al. 2020), are worth investigating too.

The food risk factors identified in the meta-analysis were corroborated by the analysis of epidemiological data. Consumption of raw eggs, undercooked pork, poultry, beef, and milk formula regularly cause outbreaks (Guillier et al., 2013; Dallman et al., 2016; Jones et al., 2016; Jourdan-da Silva et al., 2018; Dewey-Mattia et al., 2018). The data collected by the European RASFF (Rapid Alert System for Food and Feed) system also confirm the importance of these categories. Of the 170 notifications from the EU Member States, poultry meat ranks first in terms of number of alerts, followed by other meat and eggs and egg products (RASFF). Similar to Domingues et al. (2012), the consumption of dairy products was not found to be significant in the mixed population despite the frequent link between raw milk cheese consumption and Salmonella outbreaks (Yoon et al., 2016).

A potential difficulty to interpret the results of the meta-analysis could be due to the strong host specificity of some serovars (Vila-Nova et al., 2019). Indeed, the salmonellosis cases in the considered case-control studies are either caused by specific and frequently occurring serovars (i.e. Typhimurium and Enteritidis, in particular) or by many different serovars. While some serovars are not strongly associated with a unique animal reservoir (e. g. Typhimurium or Agona), others display strong associations with reservoirs, such as Enteritidis and layers, Derby and pigs, Dublin and cattle (Langridge et al., 2014). This may partly explain the variability in ORs in the different case-control studies, and the remaining heterogeneity between studies. For example, the ORs associated with egg consumption in studies focusing only on Enteritidis were likely to be higher than those from studies of other serovars. The importance of serovar in risk factor analysis has been shown recently (MacDonald et al., 2018); their results supported the notion that the epidemiology of $S$. Typhimurium may differ from other Salmonella types leading to differences in risk factors.

Besides, the relative importance of serovars in sporadic cases may differ between continents and reflect very different epidemiological situations (Ferrari et al., 2019). For example, the Heidelberg and Newport serovars are important and original in their representation in salmonellosis cases for the North American continent (Jackson et al., 2013), while they are not very important in the EU. Moreover, the different food consumption behaviors and habits in the countries where the case-control studies were carried out may have had an impact, as e.g. some kinds of meat may be mostly consumed undercooked by some, but not by others. For example, in France, the consumption of undercooked beef is a common practice (ANSES, 2017) and this consumption practice has been described as a major risk factor for Typhimurium and Enteritidis serovars (Delarocque-Astagneau et al., 2000; Delarocque-Astagneau et al., 1998). Snary et al. (2016) have shown that there is large variability concerning the type and frequency of consumption of pork products within the EU. These consumption patterns greatly impact the level of risk of pork products. On the other hand, the results of the meta-analysis conducted at the international level is very important in the context of increasing international trade in foodstuffs (Ercsey-Ravasz et al., 2012; Garre et al., 2019) and changes in food consumption patterns (Smith and Fazil, 2019). Indeed, this internationalization of trade and changes in consumer attitudes may lead to a change in risk. In this context, the identification of risk factors at the international level is, therefore, an important element for epidemiologists who often have a historical view of the different routes of transmission and specific to their country.

At this stage, the ORs cannot be used to estimate the population attributable fraction in a risk assessment. Indeed, the overall values of ORs might have been modulated by the different regulatory measures at the international level (e.g. for the table egg sector in Europe versus other continents) or within Europe (e.g. Sweden applies more stringent management measures for cattle herds than those adopted by European regulations (Ågren et al., 2015)).

The published case-control studies included this meta-analysis are stored in a database, which will be updated with other relevant studies published in the future. This opens the way for further analyses, providing a basis for comparison between different countries about the risk for salmonellosis associated with exposure to a given risk factor. Analysis of ORs observed in different countries in relation to their regulations might also indicate the appropriateness of management measures to be adopted. This basis should also make it possible to carry out various studies focusing on specific serovars, in particular Enteritidis and Typhimurium, in the future. Examination at this level would be a key element for tracing the sources of sporadic cases.

\section{Conclusion}

The results of this meta-analysis confirm the outcomes obtained in a previous systematic review of case-controls studies of salmonellosis (Domingues et al., 2012). Moreover, they help going beyond the usual suspect of egg products being always the source and stress the importance of refining the assessment of other food vehicles and consumption practices, and routes such as environmental and animal contact.

The contribution of environmental or animal contact to the risk of sporadic salmonellosis should be better documented in dedicated studies. It may be appropriate to include factors related to professional activities, and those relating to contact with pets. Finally, risk factors should be investigated by Salmonella serovars. Such investigations could enable for the identification of source-specific risk factors and underlying transmission pathways.

\section{CRediT authorship contribution statement}

Laurent Guillier: Writing - original draft. Anne Thébault: Methodology, Formal analysis, Visualization. Philippe Fravalo: Writing review \& editing. Lapo Mughini-Gras: Writing - review \& editing. Nathalie Jourdan-da Silva: Writing - review \& editing. Julie David: Writing - review \& editing. Pauline Kooh: Methodology, Project administration. Vasco Cadavez: Methodology, Investigation, Formal analysis. Ursula Gonzales-Barron: Methodology, Investigation, Formal analysis, Writing - review \& editing.

\section{Declaration of Competing Interest}

The authors declare no conflict of interest.

\section{Acknowledgments}

The authors would like to thank Anses staff and the members of the Anses Working Group on Source Attribution of Foodborne Diseases: 
Moez Sanaa, Jean Christophe Augustin, Frédéric Carlin, Alexandre Leclercq, Nicole Pavio, Isabelle Villena, and Laurence Watier. U. Gonzales-Barron and V. Cadavez are grateful to the Foundation for Science and Technology (FCT, Portugal) for financial support through national funds FCT/MCTES to CIMO (UIDB/00690/2020). U. GonzalesBarron acknowledges the national funding by FCT, P.I., through the institutional scientific employment program contract.

\section{Supplementary materials}

Supplementary material associated with this article can be found, in the online version, at doi:10.1016/j.mran.2020.100138.

\section{References}

Ågren, E.C.C., Johansson, J., Frössling, J., Wahlström, H., Emanuelson, U., SternbergLewerin, S., 2015. Factors affecting costs for on-farm control of Salmonella in Swedish dairy herds. Acta Veterinaria Scandinavica 57. https://doi.org/10.1186/ s13028-015-0118-y.

Ashbolt, R., Kirk, M.D., 2006. Salmonella Mississippi infections in Tasmania: the role of native Australian animals and untreated drinking water. Epidemiology \& Infection 134 (6), 1257-1265. https://doi.org/10.1017/S0950268806006224.

ANSES, 2017. Third national study of individual food consumption: Changes in consumption habits and patterns, new issues in the areas of food safety and nutrition. https://www.anses.fr/en/content/inca-3-changes-consumption-habits-and-patternsnew-issues-areas-food-safety-and-nutrition.

Barco, L., Barrucci, F., Olsen, J.E., Ricci, A., 2013. Salmonella source attribution based on microbial subtyping. International Journal of Food Microbiology 163 (2-3), 193-203. https://doi.org/10.1016/j.ijfoodmicro.2013.03.005.

Barco, L., Barrucci, F., Cortini, E., Ramon, E., Olsen, J.E., Luzzi, I., Lettini, A.A., Ricci, A., 2015. Ascertaining the relationship between Salmonella Typhimurium and Salmonella 4,[5],12:i:- by MLVA and inferring the sources of human salmonellosis due to the two serovars in Italy. Frontiers in Microbiology 6. https://doi.org/ 10.3389/fmicb. 2015.00301.

Bellido-Blasco, J.B., González-Cano, J.M., Galiano-Arlandis, J.V., Herrero-Carot, C., Tirado-Balaguer, M.D., Arnedo-Pena, A., Safont-Adsuara, L., Romeu-García, M.A., 2007. Risk factors for the occurrence of sporadic Campylobacter, Salmonella and rotavirus diarrhea in preschool children. Anales de pediatria 66 (4), 367-374. https://doi.org/10.1157/13101241.

Christidis, T., Hurst, M., Rudnick, W., Pintar, K.D., Pollari, F., 2020. A comparative exposure assessment of foodborne, animal contact and waterborne transmission routes of Salmonella in Canada. Food Control 109, 106899. https://doi.org/10.1016/ j.foodcont.2019.106899.

Dallman, T., Inns, T., Jombart, T., Ashton, P., Loman, N., Chatt, C., Messelhaeusser, U., Rabsch, W., Simon, S., Nikisins, S., Bernard, H., le Hello, S., Jourdan-da-Silva, N., Kornschober, C., Mossong, J., Hawkey, P., de Pinna, E., Grant, K., Cleary, P., 2016. Phylogenetic structure of European Salmonella Enteritidis outbreak correlates with national and international egg distribution network. Microbial Genomics 2. https:// doi.org/10.1099/mgen.0.000070

David, J.M., Guillemot, D., Bemrah, N., Thebault, A., Brisabois, A., Chemaly, M., Weill, F. X., Sanders, P., Watier, L., 2013a. The Bayesian microbial subtyping attribution model: robustness to prior information and a proposition. Risk Analysis 33 (3), 397-408. https://doi.org/10.1111/j.1539-6924.2012.01877.x.

David, J.M., Sanders, P., Bemrah, N., Granier, S.A., Denis, M., Weill, F.X., Guillemot, D., Watier, L., 2013b. Attribution of the French human salmonellosis cases to the main food-sources according to the type of surveillance data. Preventive Veterinary Medicine 110 (1), 12-27. https://doi.org/10.1016/j.prevetmed.2013.02.002.

Delarocque-Astagneau, E., Bouillant, C., Vaillant, V., Bouvet, P., Grimont, P.A.D., Desenclos, J.-C., 2000. Risk Factors for the Occurrence of Sporadic Salmonella enterica serotype Typhimurium infections in children in France: a national casecontrol study. Clinical Infectious Diseases 31, 488-492. https://doi.org/10.1086 313990.

Delarocque-Astagneau, E., Desenclos, J.-C., Bouvet, P., Grimont, P.A.D., 1998. Risk factors for the occurrence of sporadic Salmonella enterica serotype Enteritidis infections in children in France: a national case-control study. Epidemiology and Infection 121, 561-567. https://doi.org/10.1017/s0950268898001460.

Devleesschauwer, B., Pires, S.M., Young, I., Gill, A., and, S.E.M., 2019. Associating sporadic foodborne illness caused by Shiga toxin-producing Escherichia coli with specific foods: a systematic review and meta-analysis of case-control studies. Epidemiology and Infection 147. https://doi.org/10.1017/s0950268819001183.

Dewey-Mattia, D., Manikonda, K., Hall, A.J., Wise, M.E., Crowe, S.J., 2018. Surveillance for foodborne disease outbreaks United States 2009-2015. MMWR. Surveillance Summaries 67, 1-11. https://doi.org/10.15585/mmwr.ss6710a1.

Domingues, A.R., Pires, S.M., Halasa, T., Hald, T., 2012. Source attribution of human salmonellosis using a meta-analysis of case-control studies of sporadic infections. Epidemiology \& Infection 140 (6), 959-969. https://doi.org/10.1017/ S0950268811002172.

EFSA, ECDC, 2018. The European Union summary report on trends and sources of zoonoses, zoonotic agents and food-borne outbreaks in 2017. Efsa Journal 16.

Ercsey-Ravasz, M., Toroczkai, Z., Lakner, Z., Baranyi, J., 2012. Complexity of the international agro-food trade network and its impact on food safety. PLoS ONE 7, e37810. https://doi.org/10.1371/journal.pone.0037810.
Ferrari, R.G., Rosario, D.K.A., Cunha-Neto, A., Mano, S.B., Figueiredo, E.E.S., ConteJunior, C.A., 2019. Worldwide epidemiology of Salmonella serovars in animal-based foods: a meta-analysis. Applied and Environmental Microbiology 85. https://doi. org/10.1128/aem.00591-19.

Garre, A., Fernandez, P.S., Brereton, P., Elliott, C., Mojtahed, V., 2019. The use of trade data to predict the source and spread of food safety outbreaks: An innovative mathematical modelling approach. Food Research International 123, 712-721. https://doi.org/10.1016/j.foodres.2019.06.007.

Glass, K., Fearnley, E., Hocking, H., Raupach, J., Veitch, M., Ford, L., Kirk, M.D., 2015. Bayesian source attribution of salmonellosis in South Australia. Risk Analysis 36, 561-570. https://doi.org/10.1111/risa.12444.

Gonzales-Barron, U., Thébault, A., Kooh, P., Watier, L., Sanaa, M., Cadavez, V., 2019. Strategy for systematic review of observational studies and meta-analysis modelling of risk factors for sporadic foodborne diseases. Microbial Risk Analysis, 100082. https://doi.org/10.1016/j.mran.2019.07.003.

Guillier, L., Danan, C., Bergis, H., Delignette-Muller, M.-L., Granier, S., Rudelle, S., Beaufort, A., Brisabois, A., 2013. Use of quantitative microbial risk assessment when investigating foodborne illness outbreaks: The example of a monophasic Salmonella Typhimurium 4,5,12:i:- outbreak implicating beef burgers. International Journal of Food Microbiology 166, 471-478. https://doi.org/10.1016/j. ijfoodmicro.2013.08.006.

Guillier, L., Gourmelon, M., Lozach, S., Cadel-Six, S., Vignaud, M.-L., Munck, N.S., Hald, T., Palma, F., 2020. AB_SA: Accessory genes-Based Source Attribution - tracing the source of Salmonella enterica Typhimurium environmental strains. Microbial Genomics. https://doi.org/10.1099/mgen.0.000366.

Havelaar, A.H., Galindo, Ángela Vargas, Kurowicka, D., Cooke, R.M., 2008. Attribution of foodborne pathogens using structured expert elicitation. Foodborne Pathogens and Disease 5, 649-659. https://doi.org/10.1089/fpd.2008.0115.

Hoelzer, K., Switt, A.M., Wiedmann, M., 2011. Animal contact as a source of human nontyphoidal salmonellosis. Veterinary Research 42, 34. https://doi.org/10.1186/12979716-42-34.

IFSAC (Interagency Food Safety Analytics Collaboration), 2019. Foodborne illness source attribution estimates for 2017 for Salmonella, Escherichia coli 0157, Listeria monocytogenes, and Campylobacter using multi-year outbreak surveillance data, United States. GA and D.C.: U.S. Department of Health and Human Services. CDC, FDA,USDA-FSIS. https://www.cdc.gov/foodsafety/ifsac/pdf/P19-2017-report-Tr iAgency-508.pdf.

Inns, T., Lane, C., Peters, T., Dallman, T., Chatt, C., McFarland, N., Crook, P., Bishop, T., Edge, J., Hawker, J., Elson, R., Neal, K., Adak, G.K., Cleary, P., 2015. A multi-country Salmonella Enteritidis phage type 14b outbreak associated with eggs from a German producer: 'near real-time' application of whole genome sequencing and food chain investigations United Kingdom. May to September 2014 Eurosurveillance 20. https://doi.org/10.2807/1560-7917.es2015.20.16.21098.

Jackson, B.R., Griffin, P.M., Cole, D., Walsh, K.A., Chai, S.J., 2013. Outbreak-associated Salmonella enterica serotypes and food commodities United States, 1998-2008. Emerging Infectious Diseases 19, 1239-1244. https://doi.org/10.3201/ eid1908.121511.

Johnson, L.R., Gould, L.H., Dunn, J.R., Berkelman, R., Mahon, B.E., 2011. Salmonella infections associated with international travel: A Foodborne Diseases Active Surveillance Network (FoodNet) Study. Foodborne Pathogens and Disease 8 (9), 1031-1037.

Jones, G., Pihier, N., Vanbockstael, C., Hello, S.L., Six, S.C., Fournet, N., Jourdan-da Silva, N, 2016. Outbreak of Salmonella Enteritidis linked to the consumption of frozen beef burgers received from a food bank and originating from Poland: northern France December 2014 to April 2015. Eurosurveillance 21. https://doi.org/ 10.2807/1560-7917.es.2016.21.40.30363.

Jourdan-da Silva, N., Fabre, L., Robinson, E., Fournet, N., Nisavanh, A., Bruyand, M., Mailles, A., Serre, E., Ravel, M., Guibert, V., Issenhuth-Jeanjean, S., Renaudat, C., Tourdjman, M., Septfons, A., de Valk, H., Le Hello, S., 2018. Ongoing nationwide outbreak of Salmonella Agona associated with internationally distributed infant milk products, France, December 2017. Eurosurveillance 23 (2). https://doi.org/ 10.2807/1560-7917.ES.2018.23.2.17-00852.

Lambertini, E., Buchanan, R.L., Narrod, C., Ford, R.M., Baker, R.C., Pradhan, A.K., 2016. Quantitative assessment of human and pet exposure to Salmonella associated with dry pet foods. International Journal of Food Microbiology 216, 79-90. https://doi. org/10.1016/j.ijfoodmicro.2015.09.005.

Langridge, G.C., Fookes, M., Connor, T.R., Feltwell, T., Feasey, N., Parsons, B.N., SethSmith, H.M.B., Barquist, L., Stedman, A., Humphrey, T., Wigley, P., Peters, S.E., Maskell, D.J., Corander, J., Chabalgoity, J.A., Barrow, P., Parkhill, J., Dougan, G., Thomson, N.R., 2014. Patterns of genome evolution that have accompanied host adaptation in Salmonella. Proceedings of the National Academy of Sciences 112 863-868. https://doi.org/10.1073/pnas.1416707112.

MacDonald, E., White, R., Mexia, R., Bruun, T., Kapperud, G., Brandal, L.T., Lange, H., Nygård, K., Vold, L., 2018. The role of domestic reservoirs in domestically acquired Salmonella infections in Norway: epidemiology of salmonellosis 2000-2015, and results of a national prospective case-control study, 2010-2012. Epidemiology and Infection 147. https://doi.org/10.1017/s0950268818002911.

Mikkelä, A., Ranta, J., Tuominen, P., 2019. A modular bayesian Salmonella source attribution model for sparse data. Risk Analysis. https://doi.org/10.1111/ risa. 13310.

Mughini-Gras, L., Franz, E., van Pelt, W., 2018. New paradigms for Salmonella source attribution based on microbial subtyping. Food Microbiology 71, 60-67. https://doi. org/10.1016/j.fm.2017.03.002.

Painter, J.A., Hoekstra, R.M., Ayers, T., Tauxe, R.V., Braden, C.R., Angulo, F.J., Griffin, P. M., 2013. Attribution of foodborne illnesses, hospitalizations, and deaths to food 
commodities by using outbreak data, United States, 1998-2008. Emerging infectious diseases 19 (3), 407. https://doi.org/10.3201/eid1903.111866.

Passaro, D.J., Reporter, R., Mascola, L., Kilman, L., Malcolm, G.B., Rolka, H., Werner, S. B., Vugia, D.J., 1996. Epidemic Salmonella Enteritidis infection in Los Angeles county, California. The predominance of phage type 4 . Western journal of medicine 165 (3), 126.

Pires, S.M., Vigre, H., Makela, P., Hald, T., 2010. Using outbreak data for source attribution of human salmonellosis and campylobacteriosis in Europe. Foodborne pathogens and disease 7 (11), 1351-1361. https://doi.org/10.1089/fpd.2010.0564.

Pires, S.M., Vieira, A.R., Hald, T., Cole, D., 2014. Source attribution of human salmonellosis: an overview of methods and estimates. Foodborne pathogens and disease 11 (9), 667-676. https://doi.org/10.1089/fpd.2014.1744.

Pires, S.M., Evers, E.G., van Pelt, W., Ayers, T., Scallan, E., Angulo, F.J., Havelaar, A., Hald, T., 2009. Attributing the human disease burden of foodborne infections to specific sources. Foodborne Pathogens and Disease 6, 417-424. https://doi.org/ 10.1089/fpd.2008.0208.

RASFF 2017. The Rapid Alert System for Food and Feed - Annual Report 2016. https ://ec.europa.eu/food/sites/food/files/safety/docs/rasff_annual_report_2016.pdf.

Smith, B.A., Fazil, A., 2019. How will climate change impact microbial foodborne disease in Canada? Canada. Communicable Disease Report 45, 108-113. https://doi.org/ 10.14745/ccdr.v45i04a05.

Snary, E.L., Swart, A.N., Simons, R.R.L., Domingues, A.R.C., Vigre, H., Evers, E.G. Hald, T., Hill, A.A., 2016. A Quantitative Microbiological Risk Assessment for Salmonella in Pigs for the European Union. Risk Analysis 36, 437-449. https://doi. org/10.1111/risa.12586.

Teunis, P.F., Kasuga, F., Fazil, A., Ogden, I.D., Rotariu, O., Strachan, N.J., 2010. Dose-response modeling of Salmonella using outbreak data. International journal of food microbiology 144 (2), 243-249. https://doi.org/10.1016/j. ijfoodmicro.2010.09.026.

Ung, A., Baidjoe, A.Y., Cauteren, D.V., Fawal, N., Fabre, L., Guerrisi, C., Danis, K., Morand, A., Donguy, M.-P., Lucas, E., Rossignol, L., Lefèvre, S., Vignaud, M.-L.,
Cadel-Six, S., Lailler, R., Jourdan-da Silva, N, Hello, S.L, 2019. Disentangling a complex nationwide Salmonella Dublin outbreak associated with raw-milk cheese consumption France, 2015 to 2016. Eurosurveillance 24. https://doi.org/10.2807/ 1560-7917.es.2019.24.3.1700703.

Trepka, M.J., Archer, J.R., Altekruse, S.F., Proctor, M.E., Davis, J.P., 1999. An increase in sporadic and outbreak-associated Salmonella Enteritidis infections in Wisconsin: the role of eggs. The Journal of infectious diseases 180 (4), 1214-1219. https://doi.org/ 10.1086/314984.

Van Cauteren, D., Strat, Y.L., Sommen, C., Bruyand, M., Tourdjman, M., Jourdan-da Silva, N., Couturier, E., Fournet, N., de Valk, H., Desenclos, J.-C., 2017. Estimated annual numbers of foodborne pathogen-associated illnesses, hospitalizations, and deaths, France, 2008-2013. Emerging Infectious Diseases 23, 1486-1492. https:// doi.org/10.3201/eid2309.170081.

Van Cauteren, D., Valk, H.D., Sommen, C., King, L.A., Jourdan-da Silva, N, Weill, F.-X, Hello, S.L, Mégraud, F, Vaillant, V, Desenclos, J.C, 2015. Community incidence of campylobacteriosis and non-typhoidal salmonellosis France, 2008-2013. Foodborne Pathogens and Disease 12, 664-669. https://doi.org/10.1089/fpd.2015.1964.

Viechtbauer, W., 2010. Conducting meta-analyses in R with the metaphor package. Journal of Statistical Software 36. https://doi.org/10.18637/jss.v036.i03.

Vila-Nova, M., Durimel, K., La, K., Felten, A., Bessières, P., Mistou, M.-Y., Mariadassou, M., Radomski, N., 2019. Genetic and metabolic signatures of Salmonella enterica subsp. enterica associated with animal sources at the pangenomic scale. BMC genomics 20 (1), 814. https://doi.org/10.1186/s12864-019-6188-x.

Yoon, Y., Lee, S., Choi, K.H., 2016. Microbial benefits and risks of raw milk cheese. Food Control 63, 201-215. https://doi.org/10.1016/j.foodcont.2015.11.013.

Zhang, S., Li, S., Gu, W., den Bakker, H., Boxrud, D., Taylor, A., Roe, C., Driebe, E., Engelthaler, D.M., Allard, M., Brown, E., McDermott, P., Zhao, S., Bruce, B.B., Trees, E., Fileds, P.I., Deng, X., 2019. Zoonotic source attribution of Salmonella enterica serotype Typhimurium using genomic surveillance data. United States. Emerging infectious diseases 25 (1), 82. https://doi.org/10.3201/eid2501.180835. 